\title{
Averaged evoked potentials to classes of visual stimuli
}

H. WEINBERG AND R. E. COLE, DEPARTMENT OF PSYCHOLOGY, SIMON FRASER UNIVERSITY, Burnaby 2, British Columbia, Canada

Evoked potentials were averaged from different visual stinuli within the same class. Classes of stimuli were: Taboo words, Neutral words, Letters, and Blank flashes. Stimuli within classes were randomized and sequence of classes balanced within a group of six male and six female Ss. Estimates of spectral components showed that the frequencies 2-16 cps contributed significantly more to the averaged evoked response recorded from the class of neutral stimuli than it did any of the other classes when compared with all other classes. Differences between spectral components of other stimulus classes (Taboo, Letters, \& Blank) were not significant. An interpretation of the results involving active suppression of the effects of Taboo words is discussed.

In recent years there has been considerable interest in the question of whether different parameters of the averaged evoked response reflect coded representation of peripheral stimuli which evoke the response. Several studies have been done which do suggest that differences in complex stimuli are associated with changes in the averaged evoked response. Larsson (1960) using somatosensory stimuli, David (1964) using auditory stimuli, Chapman (1964), Lifshitz (1966) and Cohen et al (1966), all using meaningful complex visual stimuli have reported changes in the evoked response which appear to be related to differences in meaning of the stimulus.

The question of whether evoked responses reflect meaning of the stimulus is related to the work suggesting that changes in conditioned stimulus (Hearst et al, 1960; Roitbak, 1959; 1960; and Fleming, 1967).

The present study was designed to provide additional data bearing on the question of whether evoked potentials code the meaning of visual stimuli. In this study evoked potentials were averaged for classes of stimuli to determine if the response evoked by several different stimuli within a class, when averaged, would be discriminably different from the averaged response to different stimuli within another class. The two obviously different classes which were of primary interest were called "taboo words" and "neutral words."

Subjects. Six male and six female university students ranging in age between 18 and 30 were used as Ss. All had 20/20 vision either uncorrected or corrected with contact lenses.

Apparatus. The Ss were seated comfortably in a Faraday enclosure. A 3-channel tachistoscope (Scientific Prototype) was located outside the enclosure and used to present stimuli. An extension of the viewing aperature protruded through the enclosure extending the viewing distance to $1.2 \mathrm{~m}$. Evoked potentials were recorded from both right and left occipital sites using bipolar electrodes over each hemisphere. Medial and lateral electrodes on right and left sides were approximately $2.5 \mathrm{~cm}$ above the inion; medial electrodes were $2.5 \mathrm{~cm}$ and lateral electrodes $5.0 \mathrm{~cm}$ lateral to the midline.

Electrical signals were preamplified with a Grass polygrapl (Model 7) and treated by the signal averager. The instrument used for averaging evoked potentials was a 4-channel Fabri-tek. Signals were averaged at 256 addresses per channel and automatically digitized. Duration of the sweep was $512 \mathrm{msec}$ triggered by onset of the stimulus; the duration of averaging time at each address was $20 \mathrm{msec}$. Duration of stimulus presentation was $50 \mathrm{msec}$.

Twelve "emotional" and "neutral" words were chosen from Dodwell's list (1964); six in each category comprised the taboo and neutral classes. In addition, three stimulus transparencies each containing four replications of a single letter ( $L, T$, or $N$ ) were used for a nonmeaningful class called "letters." Blank flashes comprised a fifth category of stimuli called "blank."

Procedure. Four categories of stimuli were used: B, blank flashes; T, taboo words; N, neutral words: L, letters. Sixty-four presentations of blank flashes began the sequence of conditions in all sessions. This condition was followed by 64 presentations of stimuli in each of the $\mathrm{N}, \mathrm{L}$, and $\mathrm{T}$ categories: the order of N. L, and T catcgories for both males and females. Presentation of individual stimulus words within categories was randomized for each $\mathbf{S}$. Presentation of 64 blank flashes always ended the sequence of conditions in each session. Averaged evoked potentials were digitized and photographed after each category of stimuli had been presented; this involved approximately a $5 \mathrm{~min}$ rest period between categories.

Results and Discussion. In the case of several Ss it was apparent that the evoked response from right and left hemispheres was not equally clear. We decided to select for analysis the averaged evoked response of one hemisphere for each $S$. The method used for selection was to determine which hemisphere responded to the initial condition of blank flashes with the greatest amplitude of averaged deflection within the first $100 \mathrm{msec}$ after onset of the stimulus.

The averaged evoked potentials were digitized at 256 addresses (intervals of $20 \mathrm{msec}$ ). A program for estimation of spectral components over a range of 25 lags $(0-16 \mathrm{cps})$ was used with the IBM 360-40. Mean density of spectral components for all Ss were determined for each condition (Fig. 1). Using the mean density of estimated frequency for each of the frequencies $2-16 \mathrm{cps}$, $T$ tests were computed to determine differences between conditions. Conditions $\mathrm{B}, \mathrm{L}$, and $\mathrm{T}$ were all significantly different from the $\mathrm{N}$ condition $(<.01$ in each case). None of the conditions, B, L, and $\mathrm{T}$ were significantly different from each other ( $>.05$ in each case). Thus the accumulated power of the averaged evoked response accounted for by frequencies $2-16$ is about equal under conditions of B, L, T. However, these frequencies contribute to the evoked response to a significantly greater extent when resulting from the $\mathrm{N}$ condition than they do when occurring in response to the $\mathrm{B}, \mathrm{L}$,

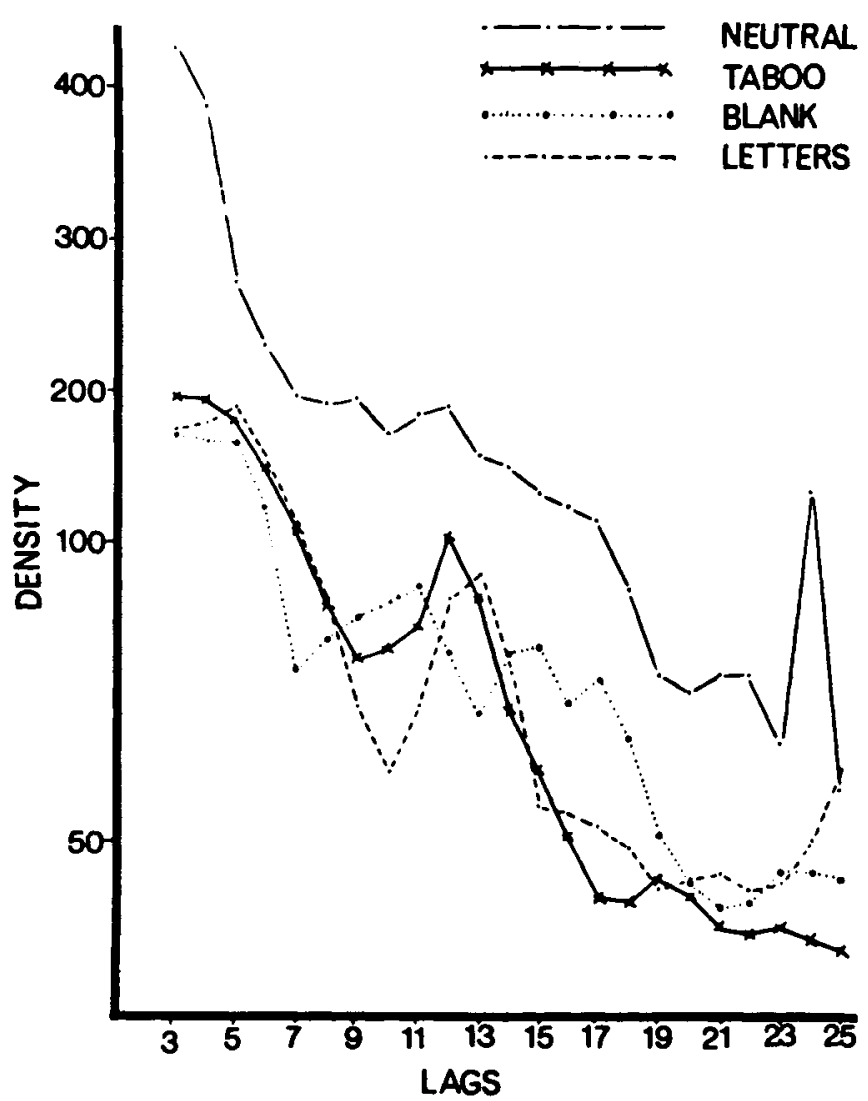

Fig. 1. Mean spectral densities for classes of stimuli. Frequency in cps equals $50 /$ no. lags. 
B

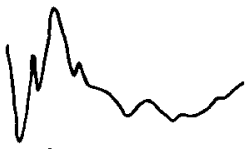

N
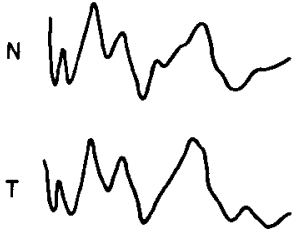

L $W V V_{\Gamma}$

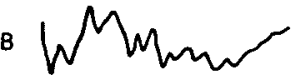

5uv

$512 \mathrm{msec}$

Fig. 2. Averaged evoked responses for S C. C.-B, blank flashes N, neutral words; $T$, taboo words; $L$, letters. Each trace is the average of 64 responses.

or $\mathrm{T}$ conditions. Further, an examination of Fig. 1 suggests that the differences between neutral and other conditions is present throughout the range of frequency components analyzed, although differences appear to be greater within the range of higher frequencies.

If these differences reflect differences in the classes of stimuli used the question arises as to why the taboo class stimuli did not result in evoked potentials which were different from either blank stimuli or letters. One interpretation which may be suggested is that some form of active inhibition is occurring such that taboo words tend to be registered in the manner of relatively meaningless stimuli like blank stimuli or letters. However, all $S$ s were required to call out the stimuli (with the exception of blank stimuli), thus insuring "perception" of the stimuli. This discrepancy could be explained if the latency of the verbal response was sufficiently longer than the latency of the evoked cortical response. If this were the case the evoked response would be more likely to reflect an initial inhibition which is overcome during the period between $500 \mathrm{msec}$ and latency of the verbal response. It is clear that differences between $\mathrm{N}$ and other conditions cannot be attributed to the requirement of a verbal response for there were no differences between $B$ condition, when verbalization was not required, and either the $L$ or the $T$ conditions when verbalization was required.

The results of this study, therefore, suggest that estimates of spectral components may differentiate between classes of neutral stimuli and taboo stimuli. The basis for this difference is, however, unclear since the relative contribution of spectral components of evoked responses to taboo words was approximately the same as was found to letters and blank flashes.

\section{REFERENCES}

CHAPMAN, R. M., \& BRAGDON, H. R. Evoked responses to numerical and non-numerical visual stimuli while problem solving. Nature, 1964, 203, $1155-1157$.

COHEN, J. \& WALTER, W. G. The interaction of responses of the brain to semantic stimuli. Psychophysiology, 1966, 2, 187-196.

DAVID, H. Enhancement of evoked cortical potentials in humans related to a task requiring a decision. Science, 1964, 182-183.

LARSSON, L. Correlation between the psychological significance of stimuli and the magnitudes of the startle blink and evoked EEG potentials in man. Acta Physiol. Scand., 1960, 48, 276-294.

LIFSHITZ, K. The averaged cortical response to complex visual stimuli. Psychophysiol., 1966, 3, 55-58.

ROITBAK, A. I. Electrical phenomena in the cerebral cortex during extinction and conditioned reflexes. EEG Clin. Neurophysiol., 1960, Suppl., 13, 91-100. 